\title{
Corrigendum: [A Comparative Study of Adaptive Interlimb Coordination Mechanisms for Self-Organized Robot Locomotion]
}

\author{
Tao Sun ${ }^{1,2}$, Xiaofeng Xiong ${ }^{2}$, Zhendong Dai ${ }^{1}$, Dai Owaki ${ }^{3}$ and Poramate Manoonpong ${ }^{1,2 *}$ \\ ${ }^{1}$ Institute of Bioinspired Structure and Surface Engineering, College of Mechanical and Electrical Engineering, Nanjing University of \\ Aeronautics and Astronautics, Nanjing, China, ${ }^{2}$ Embodied Artificial Intelligence and Neurorobotics Laboratory, SDU Biorobotics, \\ The Mærsk Mc-Kinney Møller Institute, University of Southern Denmark, Odense, Denmark, ${ }^{3}$ Department of Robotics, Graduate \\ School of Engineering, Tohoku University, Sendai, Japan
}

Keywords: adaptive interlimb coordination, phase resetting, phase modulation, decoupled CPGs, sensory feedback, self-organized locomotion

\section{OPEN ACCESS}

Approved by: Frontiers in Robotics and Al Editorial Office, Frontiers Media SA, Switzerland

${ }^{*}$ Correspondence: Poramate Manoonpong poma@mmmi.sdu.dk

Specialty section: This article was submitted to Computational Intelligence in Robotics, a section of the journal Frontiers in Robotics and $A$

Received: 29 April 2021 Accepted: 10 May 2021 Published: 02 June 2021

Citation:

Sun T, Xiong X, Dai Z, Owaki D and Manoonpong $P$ (2021) Corrigendum:

[A Comparative Study of Adaptive Interlimb Coordination Mechanisms for

Self-Organized Robot Locomotion]. Front. Robot. Al 8:702167.

doi: 10.3389/frobt.2021.702167
A corrigendum on

A Comparative Study of Adaptive Interlimb Coordination Mechanisms for Self-Organized Robot Locomotion

by Sun T, Xiong X, Dai Z, Owaki D and Manoonpong P (2021) A Comparative Study of Adaptive Interlimb Coordination Mechanisms for Self-Organized Robot Locomotion. Front. Robot. AI 8:638684. doi:10.3389/frobt.2021.638684

In the original article, there were errors.

In the published article, there was an error in affiliation 1. Instead of "College of Mechanical and Electrical Engineering, Institute of Bio-inspired Structure and Surface Engineering, Nanjing University of Aeronautics and Astronautics, Nanjing, China", it should be "Institute of Bioinspired Structure and Surface Engineering, College of Mechanical and Electrical Engineering, Nanjing University of Aeronautics and Astronautics, Nanjing, China".

In the published article, there was an error in affiliation 2. Instead of "Embodied Artificial Intelligence and Neurobotics Lab, University of Southern Denmark Biorobotics, Mærsk McKinney Møller Institute, University of Southern Denmark, Odense, Denmark", it should be "Embodied Artificial Intelligence and Neurorobotics Laboratory, SDU Biorobotics, The Mærsk Mc-Kinney Møller Institute, University of Southern Denmark, Odense, Denmark".

The authors apologize for this error and state that this does not change the scientific conclusions of the article in any way. The original article has been updated.

Copyright (c) 2021 Sun, Xiong, Dai, Owaki and Manoonpong. This is an open-access article distributed under the terms of the Creative Commons Attribution License (CC BY). The use, distribution or reproduction in other forums is permitted, provided the original author(s) and the copyright owner(s) are credited and that the original publication in this journal is cited, in accordance with accepted academic practice. No use, distribution or reproduction is permitted which does not comply with these terms. 